\title{
Molecular epidemiology of force of infection in malaria
}

\author{
Ingrid Felger ${ }^{1 *}$, Sonja Schoepflin ${ }^{1}$, Benson Kiniboro², Peter A Zimmerman³ ${ }^{3}$ Ivo Mueller ${ }^{2}$ \\ From Parasite to Prevention: Advances in the understanding of malaria \\ Edinburgh, UK. 20-22 October 2010
}

\section{Background}

Genotyping Plasmodium falciparum parasites in longitudinal studies provided a new option to estimate force of infection (FOI). FOI, defined as the number of new P.falciparum clones acquired over time, is a molecular parameter equally suitable for describing basic malaria epidemiology and measuring outcomes of clinical trials of antimalarial interventions. We investigated the ability of molecular parameters to explain differences in the risk of $P$. falciparum infection and disease between wet and dry seasons, among age groups and with respect to insecticide-treated mosquito net use.

\section{Methods}

264 children between one and three years of age from Papua New Guinea were followed over 16 months with active detection of infection at two-monthly intervals and during episodes of febrile illness. A polymerase chain reaction (PCR) for the highly polymorphic genotyping marker merozoite surface antigen 2 was performed in all blood samples. To track individual parasite clones in consecutive blood samples with maximal resolution PCR fragments were sized by capillary electrophoresis.

\section{Results}

FOI was moderately age-dependent with children under two acquiring less new $P$. falciparum clones than older ages. FOI was significantly correlated to incidence of episodes, irrespective of whether a parasite density cut off was applied or not. Seasonal variation was observed in FOI and thus risk of illness. FOI was significantly higher during the rainy season (7.47 children/year) than in the dry season $(4.30$ children/year, $\mathrm{p}<0.001)$.
Following the high transmission season the risk of clinical illness was significantly lower than after a period of low transmission.

\section{Conclusions}

Our analyses suggest a central role of molecularly determined FOI for explaining differences in the burden of clinical P. falciparum malaria in our cohort. FOI almost completely explained spatial variation, age trends and effect of bed net use on incidence. Acquisition of new parasite clones seems to be a major factor for clinical illness in these children. This study highlights the suitability of a new parameter, molecular FOI, for understanding the epidemiology of clinical malaria in young children. We propose to apply the molecular determined parameter FOI for monitoring effects of malaria interventions.

\section{Author details \\ ${ }^{1}$ Swiss Tropical and Public Health Institute, Socinstr. 57, 4002-Basel, Switzerland. ${ }^{2}$ Papua New Guinea Institute of Medical Research, PO Box 60, Goroka, Eastern Highland Province 441, Papua New Guinea. ${ }^{3}$ Center for Global Health and Diseases, Case Western Reserve University, Cleveland, $\mathrm{OH}$ 44106-7286, USA.}

Published: 20 October 2010

doi:10.1186/1475-2875-9-S2-014

Cite this article as: Felger et al:: Molecular epidemiology of force of infection in malaria. Malaria Journal 2010 9(Suppl 2):014.

${ }^{1}$ Swiss Tropical and Public Health Institute, Socinstr. 57, 4002-Basel,

Switzerland

Full list of author information is available at the end of the article

(c) 2010 Felger et al; licensee BioMed Central Ltd. This is an open access article distributed under the terms of the Creative Commons Attribution License (http://creativecommons.org/licenses/by/2.0), which permits unrestricted use, distribution, and reproduction in any medium, provided the original work is properly cited. 